\title{
ChemComm
}

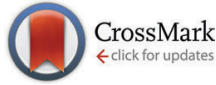

Cite this: Chem. Commun., 2015 51,8672

Received 13th March 2015,

Accepted 20th April 2015

DOI: $10.1039 / c 5 c c 02162 f$

www.rsc.org/chemcomm

\section{Intramolecular thiol-yne cyclisation as a novel strategy for thioglycal synthesis $\dagger$}

\author{
Vincent Corcé, Lauren McSweeney, Aoife Malone and Eoin M. Scanlan*
}

\begin{abstract}
A novel intramolecular thiol-yne cyclisation strategy has been developed for the synthesis of thioglycals. Both ionic and radical mediated cyclisation pathways have been investigated for $D-$ and L-sugars. The ionic cyclisation provides exclusive access to 5-exo products directly from the thioesters whereas the radical cyclisation provides access to both 5-exo and 6-endo products upon photochemical irradiation of the free thiols. These are the first examples of intramolecular thiol-yne cyclisation reactions applied to thiosugar synthesis.
\end{abstract}

Intramolecular, free-radical mediated cyclisation reactions are of critical importance for the synthesis of a diverse array of heterocycles. While many free-radical cyclisation reactions involve carbon-centred radicals, the investigation of systems where the heteroatom is the reactive radical intermediate represents an area of intensive research focus in organic chemistry. ${ }^{1}$ One such system that has received significant attention in recent times is the intramolecular thiol-ene reaction. The synthetic scope of this reaction for the efficient preparation of sulphur containing heterocycles has been extensively reviewed. ${ }^{2}$ Sulfur containing heterocycles are becoming increasingly important in the context of pharmaceutical drug design and new methods for their preparation are highly desirable. ${ }^{3}$

We have reported that the free-radical mediated, intramolecular thiol-ene reaction can be applied to the efficient synthesis of a range of thiosugars with a high degree of regioselectivity and stereoselectivity. ${ }^{4}$ In contrast to the intramolecular thiol-ene process, the related intramolecular thiol-yne reaction has not been investigated for synthetic applications. Indeed, there exists only a single report of this reaction in the literature. Surzur and co-workers investigated the cyclisation of pent-4-yne-1-thiol under photochemical conditions and observed formation of both the 5-exo and the 6-endo cyclisation

School of Chemistry, Trinity Biomedical Sciences Institute, Trinity College, 152-160 Pearse Street, Dublin 2, Ireland. E-mail: eoin.scanlan@tcd.ie;

Fax: +353 (1) 671 2896; Tel: +353 (1) 8962514

$\dagger$ Electronic supplementary information (ESI) available: Experimental procedures and analysis data for new compounds. See DOI: 10.1039/c5cc02162f products, with the six-membered ring being formed preferentially. ${ }^{5}$ We set out to investigate the intramolecular thiol-yne reaction on carbohydrate derived substrates as a novel synthetic route to both exo- and endo-thioglycals. Thioglycals are the sulphur analogues of the widely used carbohydrate building blocks, glycals and have been demonstrated to behave as competitive inhibitors of glycosidases. ${ }^{6}$ Detailed studies of their therapeutic application have been hampered by a lack of robust synthetic methodologies to prepare these substrates. Thioglycals have previously been prepared starting from commercially available 5-thio-D-glucose ${ }^{6 a}$ but this reagent is prohibitively expensive for use in multistep synthesis and offers only limited access to thioglycals. We reasoned that the intramolecular thiol-yne reaction would offer a general and efficient synthetic route to novel thioglycals and thiosugars as putative glycosidase inhibitors. The related intermolecular thiol-yne process is emerging as a widely employed conjugation reaction with applications in materials science, supramolecular chemistry and bioconjugation. ${ }^{7}$ The mild reaction conditions, high yields and tolerance of an aqueous environment have facilitated the application of the intermolecular thiol-yne reaction to the preparation of bioconjugates, including glycopeptides and glycoproteins. ${ }^{2 b, 8}$ The factors that make the intermolecular reaction attractive for bioconjugation are also relevant for the intramolecular process and should provide a high-yielding and mild strategy for accessing thioglycals.

In order to study the intramolecular thiol-yne reaction on a carbohydrate substrate, we first needed to develop a robust synthetic methodology for accessing acyclic glycans with a free thiol and terminal alkyne group suitable for intramolecular cyclisation (Fig. 1).

Starting from the commercially available $O$-benzyl protected arabinose 1, a number of strategies were investigated for introduction of the terminal alkyne at the anomeric position of the sugar. Initially, we attempted to employ Corey-Fuchs ${ }^{9}$ conditions directly at the hemiacetal, but this resulted only in the formation of degradation products. We also investigated the use of the Bestmann-Ohira reagent which has previously been applied to carbohydrates systems, albeit in low yields. The Bestmann-Ohira reagent furnished the desired product 2 in a 


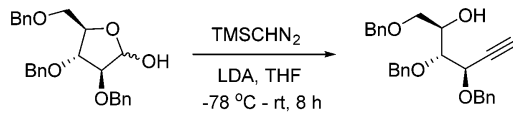

1

2, $70 \%$
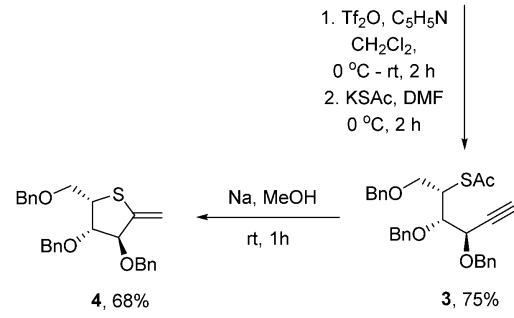

Fig. 1 Synthetic strategy for preparation of L-thioglycal.

low yield of $25 \%$ but it was inseparable from the C-2 epimerization byproduct. Finally we investigated the use of the Colvinrearrangement for introduction of the alkyne. This reaction proceeded via a lithiated trimethylsilyl diazomethane intermediate which was formed at low temperature. This method furnished the alkyne 2 in 70\% yield. Vasella and co-workers previously employed a 1-diazo-2-oxopropyl phosphonate reagent to prepare the alkyne derivative of $O$-benzyl protected D-ribose in $61 \%$ yield. ${ }^{10}$ Treatment of 2 with triflic anhydride followed by nucleophilic displacement with KSAc furnished thioester 3 in $75 \%$ over two steps. Treatment of the thioester under Zemplén conditions was carried out in an attempt to access the free thiol, but was found to promote spontaneous formation of the 5-exo-glycal product 4 cleanly in $68 \%$ yield. While the term 'thiol-yne' usually refers to the radical mediated reaction, a limited number of base-catalysed, intramolecular, ionic reactions have previously been reported, but only on disubstituted alkynes and never on carbohydrate substrates. ${ }^{11}$ In order to further investigate the scope of this cyclisation reaction, synthesis of the D-sugar was carried out following a related synthetic approach. A double inversion strategy was employed in order to introduce the desired D-sugar stereochemistry at C-5. ${ }^{4 b}$ The first inversion reaction was carried out with para-nitrobenzoic acid under Mitsunobu conditions, followed by cleavage of the resulting ester under basic conditions to furnish 5. KSAc displacement of the intermediate triflate was employed to furnish the thioester 6 (Fig. 2).

Thioester 6 was treated under Zemplén conditions and spontaneous cyclisation to the 5-exo-glycal 7 occurred in excellent yield and with complete regioselectivity. The results from both the D- and L-sugars 6 and 3 demonstrated that ionic conditions could be employed to access exo-thioglycals in high yield directly from the corresponding acylic thioester. The ionic reactions were highly regioselective towards the 5-exo products. The improvement in yield observed for the cyclisation of the D-sugar over the L-sugar may be due to steric interactions associated with the relative orientation of the bulky benzyl-protected hydroxyl groups. In the D-sugar, all of the hydroxyl groups adopt a sterically less hindered equatorial orientation which may assist the cyclisation reaction.

Although the ionic cyclisation pathway provided a novel and efficient synthetic route to 5-exo-thioglycals, we still required access to the free thiol substrates in order to investigate the thiyl-radical mediated cyclisation reaction. We therefore set out

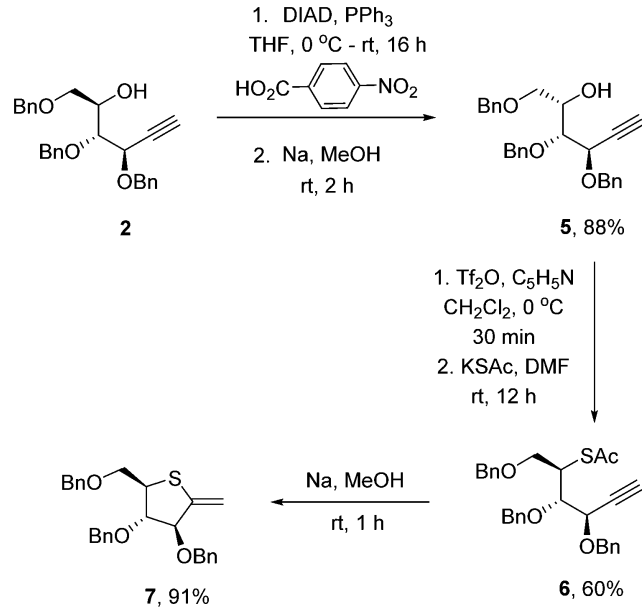

Fig. 2 Synthetic strategy for preparation of D-thioglycal.

to investigate conditions that would allow access to the thiols $\mathbf{8}$ and 9 directly from the corresponding thioesters. Synthesis of the free thiols 8 and $\mathbf{9}$ proved extremely challenging. Basic conditions could not be employed since they would result in spontaneous cyclisation to the 5-exo thioglycal. Starting from the thioester $\mathbf{6}$, a number of reaction conditions were screened in order to prepare the desired thiol 8. Attempts to use hydride sources such as $\mathrm{LiAlH}_{4}$ or $\mathrm{NaBH}_{4}$ in ethanol or diethyl ether failed to furnish any of the desired thiol. Acidic deprotection conditions employing acetyl chloride in methanol resulted in the formation of complex mixture of products including a small amount of the desired thiol 8, starting material 6 and the exoglycal 7. Encouraged by this result we screened a number of acidic deprotection conditions including the use of Dowex resin in methane thiol and in methanol. In each case, only starting material 6 was obtained. Use of stoichiometric equivalents of the nucleophilic amines, phenyl hydrazine and dinitrophenyl hydrazine also resulted in only starting material 6 being recovered. Finally we investigated the deacetylation reaction with excess $\mathrm{MeLi}$ as a reagent and this furnished the desired thiol 8 in 50\% yield. A three-fold excess of MeLi was required in order to access the free thiol without concomitant formation of the competing exo-product 7 . We rationalised that the intramolecular cyclisation via the thiolate is prevented by employing an excess of $\mathrm{MeLi}$ that also deprotonates the terminal alkyne, thus creating an ionic intermediate where intramolecular cyclisation is highly disfavoured due to the electronic repulsion between the anion pair. Although the conditions employed for the release of the thiol are relatively harsh for thioester deprotection, these were the only conditions found under which the free thiol 8 could be accessed in a synthetically useful yield. The unreacted thioester 6 was the only other product recovered from this reaction and this could be reused for thiol preparation. Identical conditions were applied to the L-sugar 3 to furnish the thiol $\mathbf{9}$. The thiols 8 and 9 were unstable at room temperature and decomposed to a range of products upon standing for several hours. They could however be maintained at $-20{ }^{\circ} \mathrm{C}$ under argon for several days before decomposition occurred (Fig. 3). 


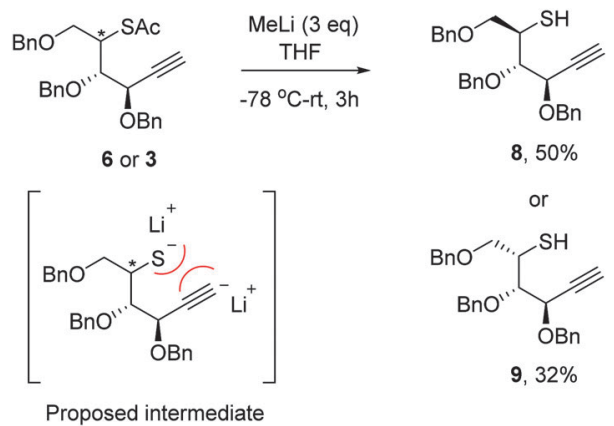

Fig. $\mathbf{3}$ Preparation of free thiols $\mathbf{8}$ and $\mathbf{9}$ from thioesters $\mathbf{6}$ and $\mathbf{3}$.
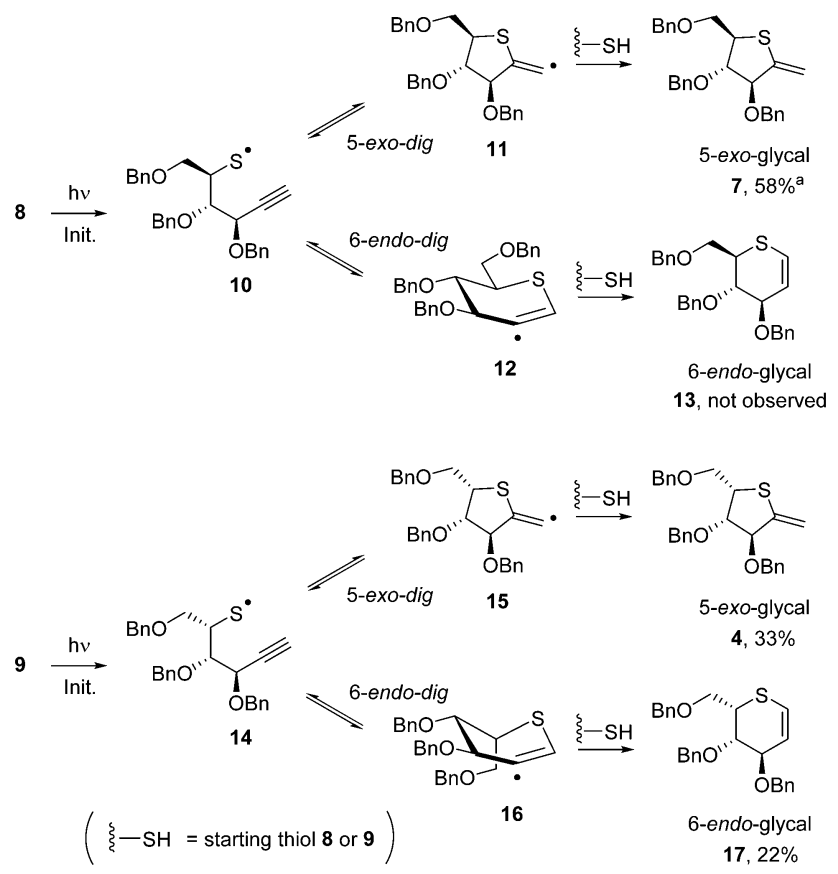

Fig. 4 Product distribution observed on treatment of $\mathbf{8}$ and $\mathbf{9}$ under photochemical initiation conditions. ${ }^{a}$ Yield determined by ${ }^{1} \mathrm{H}$-NMR relative to unreacted starting material $\mathbf{8}$.

Following the successful preparation of the radical precursors 8 and 9, the intramolecular free-radical mediated thiol-yne cyclisation reaction was investigated. The radical reactions were carried out in a degassed solution of DMF using 2,2-dimethoxy2-phenyl-acetophenone (DPAP) as an initiator and 2-methylbenzophenone (MBP) as a photosensitiser. The reaction conditions used were those previously optimised for the related thiol-ene reaction. ${ }^{4 a}$ For each substrate, the reaction mixture was irradiated under UV for one hour at rt without agitation. The cyclisation pathway and product distribution for both D- and L-sugars are illustrated in Fig. 4.

The results of the free-radical mediated cyclisation reaction vary significantly between the D-sugar and the L-sugar. For the D-sugar, the intramolecular thiol-yne reaction furnished the 5-exo glycal 7 exclusively as the major product. None of the corresponding 6-endo-dig product 13 was observed. The only other product observed was unreacted starting material 8. In contrast, the
L-sugar furnished a separable mixture of both the 5-exo and 6 -endo glycals in a combined yield of $55 \%$ and in a ratio of $3: 2$ in favour of the exo-product. These results are in stark contrast to those reported by Surzur for the unsubstituted pent-4-yne-1thiol substrate where 6-endo cyclisation was favoured. ${ }^{5}$ These results demonstrate that the stereochemistry of the substituents on the carbohydrate backbone has a significant influence over the regioselectivity of the cyclisation reaction in a manner analogous to the thiol-ene reaction. ${ }^{2 a}$ The nature of the protecting groups may also play an important role and these factors are currently under investigation in our group. The fact that no significant byproducts and no competing intramolecular hydrogen abstraction processes were observed is noteworthy and highlights the potential of these reactions for thiosugar synthesis.

In conclusion, we have demonstrated the very first examples of intramolecular thiol-yne cyclisation reactions for the preparation of thioglycals. The ionic pathway provides regioselective access to 5-exo-glycal products in high yield. The radical mediated process provides access to the 5-exo product exclusively when using the D-sugar and a mixture of both the 5-exo and 6-endo products for the L-sugar. Synthetic routes for the preparation of both thioesters and free-thiols suitable for cyclisation reactions have been developed. These results highlight the potential of the free radical process to provide access to 6-endo thioglycal products suitable for screening as glycosidase inhibitors. Current studies are underway to develop the full synthetic scope of this methodology for a range of carbohydrates and to explore conditions for promoting the 6-endo-dig cyclisation pathway for accessing endo-thioglycals.

\section{Notes and references}

1 (a) T. Naito, Pure Appl. Chem., 2008, 80, 717; (b) X. Xu, X. Wan, Y. Geng, J. Zhang and H. Xu, Youji Huaxue, 2011, 31, 453.

2 (a) E. M. Scanlan, V. Corce and A. Malone, Molecules, 2014, 19, 19137; (b) F. Denes, M. Pichowicz, G. Povie and P. Renaud, Chem. Rev., 2014, 114, 2587.

3 B. R. Beno, K.-S. Yeung, M. D. Bartberger, L. D. Pennington and N. A. Meanwell, J. Med. Chem., 2015, DOI: 10.1021/jm501853m.

4 (a) A. Malone and E. M. Scanlan, Org. Lett., 2013, 15, 504; (b) A. Malone and E. M. Scanlan, J. Org. Chem., 2013, 78, 10917.

5 C. Dupuy, M. P. Crozet and J. M. Surzur, Bull. Soc. Chim. Fr., 1980, 361.

6 (a) W. Korytnyk, N. Angelino, O. Dodson-Simmons, M. Hanchak, M. Madson and S. Valentekovic-Horvath, Carbohydr. Res., 1983, 113, 166; $(b)$ P. Greimel, J. Spreitz, F. K. Sprenger, A. E. Stutz and T. M. Wrodnigg, Org. Chem. Sugars, 2006, 383.

7 (a) B. C. Yao, J. Z. Sun, A. J. Qin and B. Z. Tang, Chin. Sci. Bull., 2013, 58, 2711; (b) A. B. Lowe, Polymer, 2014, 55, 5517; (c) M. Ghirardello, K. Oeberg, S. Staderini, O. Renaudet, N. Berthet, P. Dumy, Y. Hed, A. Marra, M. Malkoch and A. Dondoni, J. Polym. Sci., Part A: Polym. Chem., 2014, 52, 2422.

8 M. Lo Conte, S. Staderini, A. Marra, M. Sanchez-Navarro, B. G. Davis and A. Dondoni, Chem. Commun., 2011, 47, 11086.

9 E. J. Corey and P. L. Fuchs, Tetrahedron Lett., 1972, 3769.

$10 \mathrm{M} . \mathrm{Xu}, \mathrm{Z}$. Miao, B. Bernet and A. Vasella, Helv. Chim. Acta, 2005, 88, 2918.

11 (a) A. Kondoh, K. Takami, H. Yorimitsu and K. Oshima, J. Org. Chem., 2005, 70, 6468; (b) A. Massi and D. Nanni, Org. Biomol. Chem., 2012, 10, 3791; (c) V. K. Aggarwal, R. Angelaud, D. Bihan, P. Blackburn, R. Fieldhouse, S. J. Fonquerna, G. D. Ford, G. Hynd, E. Jones, R. V. H. Jones, P. Jubault, M. J. Palmer, P. D. Ratcliffe and H. Adams, J. Chem. Soc., Perkin Trans. 1, 2001, 2604; (d) T. Fujisawa, M. Nagai, Y. Koike and M. Shimizu, J. Org. Chem., 1994, 59, 5865. 